\title{
A 3-component system of competition and diffusion
}

\author{
Masayasu Mimura ${ }^{\dagger}$ and Paul C. FifE ${ }^{\dagger}$
}

(Received May 20, 1985)

\begin{abstract}
This report studies the existence of non-constant solutions of certain two-point boundary value problems for 3-component systems with a small parameter $\varepsilon$, under homogeneous Neumann conditions at the boundaries. This problem is related to the analysis of segregation patterns in population models of 3-competing and spatially dispersing species. It is shown that the reduced problem $(\varepsilon=0)$ has many non-constant solutions exhibiting spatial segregation. Only a few of these, however, can serve as valid lowest-order approximations to solutions of the original problem when $\varepsilon$ is non-zero but small. A singular perturbation construction clarifies which are in this category. The results of numerical computations of solutions are also illustrated.
\end{abstract}

\section{Introduction}

We consider populations of $N$ species $S_{1}, S_{2}, \ldots, S_{N}$ in a bounded habitat, and assume that the distribution of the populations are determined by competition of Lotka-Volterra-Gause type and simple diffusion. Suppose $u_{i}(t, x)$ $(i=1,2, \ldots, N)$ is the population density of the species $S_{i}(i=1,2, \ldots, N)$. The spatial domain is taken to be the one-dimensional interval $(0,1)$. Then we have the following reaction-diffusion equations governing the evolution of the $u_{i}$ :

$$
\begin{array}{r}
\frac{\partial u_{i}}{\partial t}=d_{i} \frac{\partial^{2} u_{i}}{\partial x^{2}}+\left(r_{i}-\sum_{j=1}^{N} a_{i j} u_{j}\right) u_{i}, t>0, x \in(0,1), \\
(i=1,2, \ldots, N)
\end{array}
$$

where $d_{i}, r_{i}$ and $a_{i j}(i, j=1,2, \ldots, N)$ are non-negative constants. In ecological terms, $r_{i}$ is the intrinsic growth rate of $S_{i}, a_{i i}$ is a measure of intraspecific competition of $S_{i}$, and $a_{i j}(i \neq j)$ is a measure of interspecific competition between the species. The boundary and initial conditions are taken to be

$$
\frac{\partial u_{i}}{\partial x}(t, x)=0, \quad t>0, x=0,1
$$

and

† This work was done while the authors were at the Mathematics Research Center, Madison, Wisconsin (June 1982). 


$$
u_{i}(0, x)=u_{i 0}(x) \geqq 0, \quad x \in[0,1], \quad(i=1,2, \ldots, N)
$$

The case of two competing species $(N=2)$

$$
\begin{aligned}
& \frac{\partial u_{1}}{\partial t}=d_{1} \frac{\partial^{2} u_{1}}{\partial x^{2}}+\left(r_{1}-a_{11} u_{1}-a_{12} u_{2}\right) u_{1} \\
& \frac{\partial u_{2}}{\partial t}=d_{2} \frac{\partial^{2} u_{2}}{\partial x^{2}}+\left(r_{2}-a_{21} u_{1}-a_{22} u_{2}\right) u_{2}
\end{aligned}
$$

has been studied extensively. When $r_{i}$ and $a_{i j}(i, j=1,2)$ are fixed arbitrarily except that the inequalities

$$
\frac{a_{11}}{a_{21}}<\frac{r_{1}}{r_{2}}<\frac{a_{12}}{a_{22}}
$$

are not both satisfied, the solution of (4), (2), (3) tends to a constant steady state solution (for instance, [2]). That is, there never appears any spatial segregation for large time. Furthermore, in the remaining case

$$
\frac{a_{11}}{a_{21}}<\frac{r_{1}}{r_{2}}<\frac{a_{12}}{a_{22}}
$$

Kishimoto [5] showed that even if there exist non-constant nonnegative steady state solutions, these are unstable. This result indicates that the simple diffusioncompetition model (4) cannot explain spatially segregated distributions of two competing species. The situation is different when one introduces the effect of cross-population pressure [14] into (4). It is shown in [12] that there occur non-constant steady state solutions exhibiting spatial segregation on the basis of cross-diffusion-induced instability (see also [10]).

These results motivate us to study whether or not systems with more than two components $(N \geqq 3)$ can exhibit spatial segregation. In this connection, Evans [3] and Kishimoto [6] have already presented some examples of competition and/or prey-predator systems in the case $N=3$ which show diffusion-induced instability. This phenomenon has usually been associated with activator-inhibitor systems, but competitive interaction does not fall into that category. A few examples of systems with stable non-constant bifurcating steady state solutions were given in [6].

Furthermore, Kishimoto, Mimura and Yoshida [7] have studied the system (1) with $N=4$ and have shown that there appear time-periodic, spatially nonconstant solutions.

In this paper, we will be interested in the stationary problem (1) and (2) with $N=3$ under the conditions that two of the diffusion coefficients are sufficiently small. We show the existence of non-constant, nonnegative solutions by a singular perturbation construction. 


\section{Ecological background and assumption}

Uncovering the mechanisms for spatial patterning of ecological communities has been a very active line or research in population biology. Most often, spatial diversity in connected habitats has been assumed to be linked to some heterogeneity in the environment, but it has also been recognized (Levin [9], for example) that stable patterns can exist in otherwise homogeneous environments. A celebrated mathematical model displaying such features in the analogous context of chemical interaction (and diffusion) was presented by Turing [16] in 1954, and model systems of this general type have been elaborated extensively since then.

Our context, of course, is a continuous space-time population model incorporating species interaction and dispersal. Interactions of prey-predator type are analogous to those of "activator-inhibitor" type occurring in other disciplines, and are most reminiscent of the type of chemical interaction that Turing postulated. And, in fact, small amplitude patterns for two-species prey-predator situations with spatial dispersal modelled by diffustion operators can, in many cases, be constructed by standard bifurcation techniques.

Competition-type interactions between two species are not of activatorinhibitor type, however, and so it is not too surprising that bifurcation techniques provide no stable patterns for two competing species models. Kishimoto and Weinberger [8] showed that no stable non-constant solutions of the corresponding boundary value problem (bounded and convex domain in one or more dimensions) with no-flux boundary condition can exist. To the contrary, Matano and Mimura [10] constructed stable non-constant solutions in suitable nonconvex domains. These raise the question as to whether patterns are possible in more than twospecies competition systems in convex domains.

The present paper shows that patterns are possible in three-species competition systems with diffusion. The technique used is not bifurcation, but rather formal singular perturbation layer analysis, utilizing large differences in the diffusion rates.

In practical terms, these results simply serve to point out yet another mechanism for stable spatial diversity in homogeneous environments. There has been, of course, no experimental or observational studies which clearly demonstrate the action of this mechanism in nature, although segreagtion phenomena in communities of three species, possible competing, have been studied. For example, Trägårdh [15] studies the interaction of three (and more) species of pine beetles. When Blastophagus piniperda and B. minor were present on a tree, their ranges were observed to be segregated: $B$. piniperda occupied the lower, and $B$. minor the upper region of the trunk. But on a small island $B$. minor was not seen, and the range of the other species was enlarged. A third 
species, Ips longicollis, living more in the outer part of the bark, coexisted throughout the ranges of both Blastophagus species. Crombie [1] observed a similar ecosystem with Rhizoperta dominica, Sltotroga cerealella, and Oryzaephilus surinamensis. Competition among three or more species, irrespective of spatial distribution considerations, is recently under investigation by many researchers; for a survey of some of this work, see Schoener [13].

We shall investigate three-species competition systems, and shall look for spatial distributions similar to those mentioned above, observed by Trägårdh and Crombie.

Consider the stationary version of (1) and (2) with $N=3$,

$$
\left\{\begin{array}{l}
0=d_{1} \frac{d^{2} u_{1}}{d x^{2}}+\left(r_{1}-a_{11} u_{1}-a_{12} u_{2}-a_{13} u_{3}\right) u_{1}, \\
0=d_{2} \frac{d^{2} u_{2}}{d x^{2}}+\left(r_{2}-a_{21} u_{1}-a_{22} u_{2}-a_{23} u_{3}\right) u_{2}, x \in(0,1), \\
0=d_{3} \frac{d^{2} u_{3}}{d x^{2}}+\left(r_{3}-a_{31} u_{1}-a_{32} u_{2}-a_{33} u_{3}\right) u_{3},
\end{array}\right.
$$

and the boundary conditions

$$
\frac{d u_{i}}{d x}=0, x=0,1, \quad(i=1,2,3) .
$$

We impose some conditions on $r_{i}$ and $a_{i j}(i, j=1,2,3)$;

$$
\frac{a_{22}}{a_{32}}<\frac{a_{23}}{a_{33}}<\frac{r_{2}}{r_{3}} \text {. }
$$

In ecological terms, this implies that when $S_{1}$ is absent, $S_{2}$ can survive and $S_{3}$ becomes extinct in competition. That is, when $u_{1} \equiv 0$,

$$
\lim _{t \rightarrow \infty}\left(u_{2}(t, x), u_{3}(t, x)\right)=\left(\frac{r_{2}}{a_{22}}, 0\right)
$$

(de Mottoni [2]).

$$
\frac{a_{12}}{a_{22}}<\frac{r_{1}}{r_{2}}<\frac{a_{11}}{a_{21}} \text { and } \frac{a_{13}}{a_{33}}<\frac{r_{1}}{r_{3}}<\frac{a_{11}}{a_{31}},
$$

which imply that in the absence of $S_{3}$ (resp. $S_{2}$ ), $S_{1}$ and $S_{2}$ (resp. $S_{3}$ ) may coexist in competition. (See Figure 1).

REMARK 1. The argument in this paper will also handle the case when $S_{2}$ survives to the exclusion of $S_{1}$, when $S_{3}$ is absent, the other asumptions above remaining unchanged. 


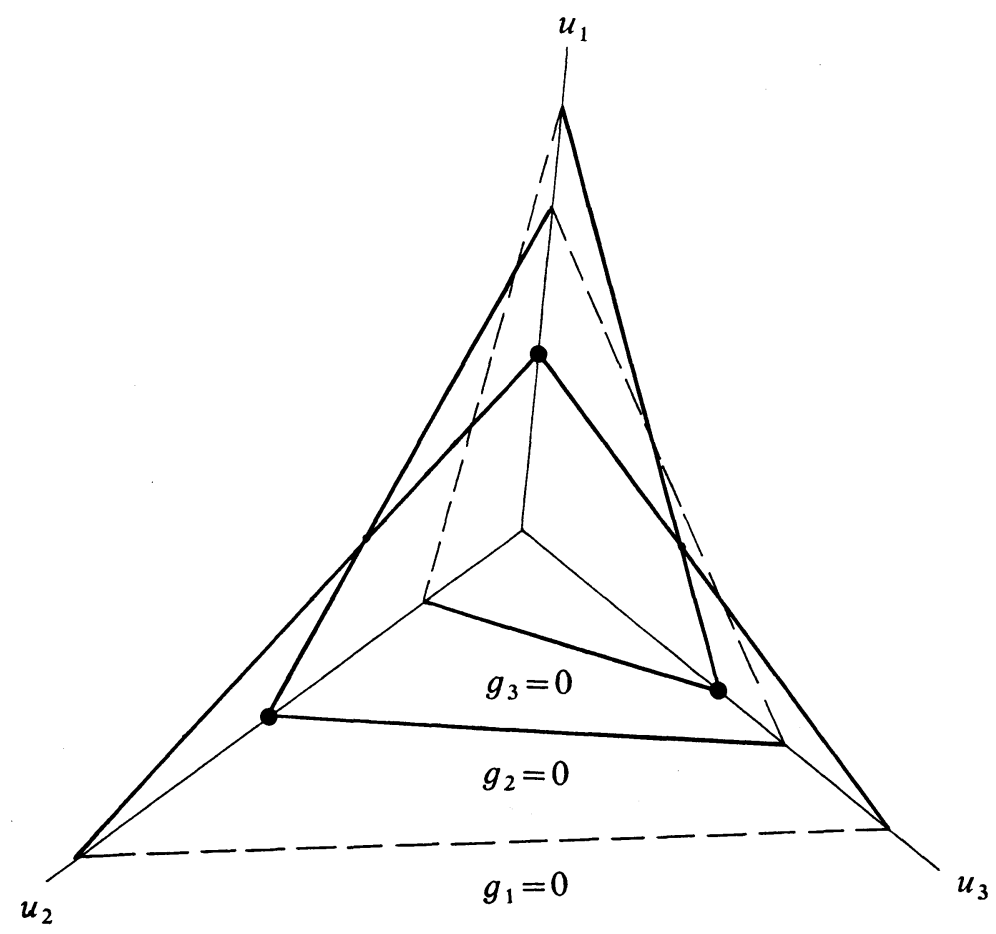

Figure 1. Location of the null planes of the dynamics $g_{i}=r_{i}-\sum_{j=1}^{3} a_{i j} u_{j}$ $(i=1,2,3)$. means the trivial critical points of $(5)$.

$$
\begin{array}{r}
S_{2} \text { and } S_{3} \text { are both slowly diffusing, that is, } d_{1}=1, d_{2}=\varepsilon^{2} \\
\text { and } d_{3}=d \varepsilon^{2} \text { for some constant } d>0(0<\varepsilon \ll 1) .
\end{array}
$$

We will impose some conditions on $r_{i}$ and $a_{i j}(i, j=1,2,3)$ in addition to (H1) and (H2) later. Note that the question as to whether all three species can coexist in the absence of diffusion is not relevant to the present study.

\section{Singular perturbation problem}

In this section, we consider the reduced system corresponding to (5):

(7)

$$
\begin{cases}0=\frac{d^{2} v_{1}}{d x^{2}}+ & \left(r_{1}-a_{11} v_{1}-a_{12} v_{2}-a_{13} v_{3}\right) v_{1}, x \in(0,1) \\ 0= & \left(r_{2}-a_{21} v_{1}-a_{22} v_{2}-a_{23} v_{3}\right) v_{2}, \\ 0= & \left(r_{3}-a_{31} v_{1}-a_{32} v_{2}-a_{33} v_{3}\right) v_{3}\end{cases}
$$

subject to the boundary conditions 


$$
\frac{d v_{1}}{d x}=0, x=0,1
$$

From the second and the third equations of (7), we have four possibilities

$$
\begin{aligned}
& \text { (I) } v_{2}=v_{3}=0, \\
& \text { (II) } v_{2}=\frac{r_{2}-a_{21} v_{1}}{a_{22}}, v_{3}=0, \\
& \text { (III) } v_{2}=0, v_{3}=\frac{r_{3}-a_{31} v_{1}}{a_{33}}
\end{aligned}
$$

and

$$
\begin{gathered}
\text { (IV) } v_{2}=\frac{\left(r_{2}-a_{21} v_{1}\right) a_{33}-\left(r_{3}-a_{31} v_{1}\right) a_{23}}{a_{22} a_{33}-a_{32} a_{23}}, \\
v_{3}=\frac{\left(r_{3}-a_{31} v_{1}\right) a_{22}-\left(r_{2}-a_{21} v_{1}\right) a_{32}}{a_{22} a_{33}-a_{32} a_{23}}
\end{gathered}
$$

Substituting the relations ( I )-(IV) into the first equation of (7), we obtain scalar equations for $v_{1}$,

$$
0=\frac{d^{2} v_{1}}{d x^{2}}+f_{i}\left(v_{1}\right), x \in(0,1) \quad(i=\mathrm{I}, \mathrm{II}, \mathrm{III}, \mathrm{IV})
$$

subject to the boundary condition (8), where $f_{i}(v)(i=\mathrm{I}, \mathrm{II}, \mathrm{III}, \mathrm{IV})$ take the forms

$$
\begin{aligned}
f_{\mathrm{I}}(v) & =\left(r_{1}-a_{11} v\right) v, \\
f_{\text {II }}(v) & =\left(\frac{r_{1} a_{22}-a_{12} r_{2}}{a_{22}}-\frac{a_{11} a_{22}-a_{12} a_{21}}{a_{22}} v\right) v, \\
f_{\text {III }}(v) & =\left(\frac{r_{1} a_{33}-a_{13} r_{3}}{a_{33}}-\frac{a_{11} a_{33}-a_{13} a_{31}}{a_{33}} v\right) v,
\end{aligned}
$$

and

$$
\begin{aligned}
& f_{\mathrm{IV}}(v)=\frac{1}{a_{22} a_{33}-a_{32} a_{23}}\left[\left(a_{22} a_{33}-a_{32} a_{23}\right) r_{1}+\left(a_{13} a_{32}-a_{12} a_{33}\right) r_{2}\right. \\
& +\left(a_{12} a_{23}-a_{13} a_{22}\right) r_{3}-\left\{a_{11}\left(a_{22} a_{33}-a_{32} a_{23}\right)+a_{12}\left(a_{31} a_{23}-a_{21} a_{33}\right)\right. \\
& \left.\left.+a_{13}\left(a_{21} a_{32}-a_{31} a_{22}\right)\right\} v\right] v .
\end{aligned}
$$

It is obvious that the forms of $f_{\mathrm{I}}, f_{\mathrm{II}}$ and $f_{\mathrm{III}}$ are all convex from above (Figure 2). Therefore, the problems $(9)_{i}(i=\mathrm{I}, \mathrm{II}, \mathrm{III})$ subject to (8) have no non-constant nonnegative solutions. (However, $(9)_{\mathrm{IV}}$ may indeed possess such solutions for suitable $r_{i}$ and $a_{i j}(i, j=1,2,3)$. Our solutions will be constructed differentily.) 


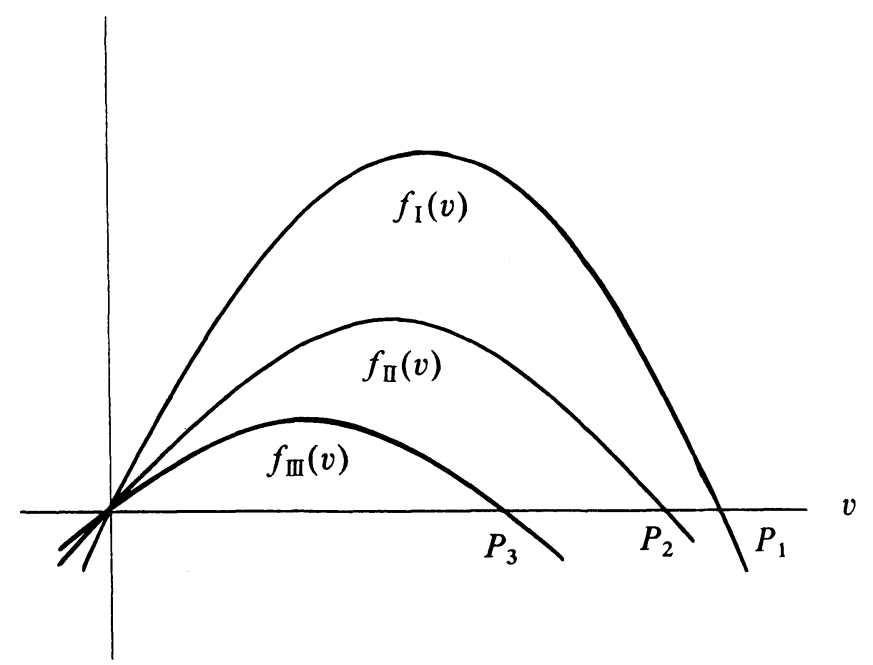

Figure 2. Functional forms of $f_{i}(v)(i=1,2,3) . \quad P_{1}=r_{1} / a_{11}, \quad P_{2}=\bar{u}_{1}(12 \backslash 3)$ and $P_{3}=\bar{u}_{1}(13 \backslash 2)$.

We now assume

$$
\bar{u}_{1}(12 \backslash 3)=\frac{r_{1} a_{22}-a_{12} r_{2}}{a_{11} a_{22}-a_{12} a_{21}} \neq \frac{r_{1} a_{33}-a_{13} r_{3}}{a_{11} a_{33}-a_{13} a_{31}}=\bar{u}_{1}(13 \backslash 2),
$$

where $\bar{u}_{1}(12 \backslash 3)$ is the first component of the solution $\left(u_{1}, u_{2}\right)$ of $r_{1}-a_{11} u_{1}-$ $a_{12} u_{2}=r_{2}-a_{21} u_{1}-a_{22} u_{2}=0 . \quad \bar{u}_{1}(13 \backslash 2)$ is similarly understood. We take

$$
\bar{u}_{1}(12 \backslash 3)>\bar{u}_{1}(13 \backslash 2) \text {. }
$$

Here we construct a new function $f_{\text {II III }}(v)$ defined by

$$
f_{\mathrm{II} \mathrm{III}}(v ; \xi)= \begin{cases}f_{\mathrm{II}}(v) & \text { for } \xi<v \\ f_{\mathrm{III}}(v) & \text { for } 0<v<\xi,\end{cases}
$$

where $\xi\left(\bar{u}_{1}(12 \backslash 3)<\xi<\bar{u}_{1}(13 \backslash 2)\right)$ is arbitrarily fixed, and consider the problem

$$
0=\frac{d^{2} v_{1}}{d x^{2}}+f_{\text {II III }}\left(v_{1} ; \xi\right), x \in(0,1)
$$

subject to the boundary condition (8).

THEOREM 1. Fix $\xi$ arbitrarily so that $\max \left(\bar{u}_{1}(13 \backslash 2), \frac{1}{2} \bar{u}_{1}(12 \backslash 3)\right)<\xi<$ $\bar{u}_{1}(12 \backslash 3)$. Then the problem (10), (8) has a non-constant nonnegative solution $v_{1}(x ; \xi)$ satisfying 


$$
\bar{u}_{1}(13 \backslash 2)<v_{1}(x ; \xi)<\bar{u}_{1}(12 \backslash 3) .
$$

Proof: See Mimura [11]. follows:

Using this function $v_{1}(x ; \xi)$, we obtain a solution of the problem (7), (8) as

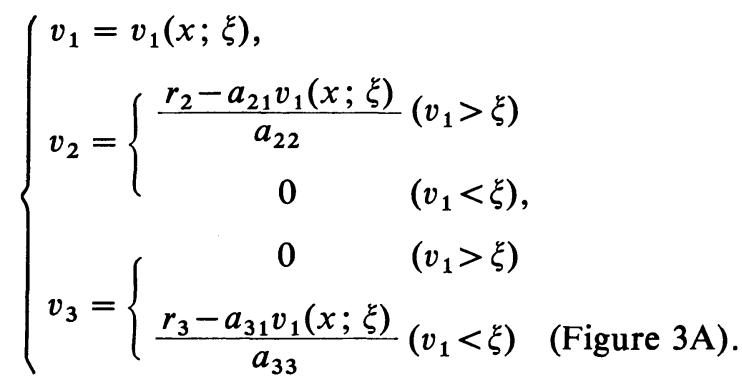

The solution (11) shows that $S_{2}$ and $S_{3}$ are coexisting with spatial structure of segregation. This is an interesting phenomenon because, in the absence of $S_{1}$, $S_{3}$ always becomes extinct under the assumption (H1). That is, the presence of $S_{1}$ makes the coexistence of $S_{2}$ and $S_{3}$ possible.

We next consider the problem of whether the solution of the reduced problem (11) becomes a lowest order approximation to a solution of the original problem (5) when $\varepsilon$ is sufficiently small but non-zero. The solution will be sought in separate regions named "inner" and "outer" regions and then matched appropriately. It is expected that the function (11) becomes an "outer" solution outside a neighborhood of $x=x^{*}$, where $x^{*}$ is a point where $v_{1}\left(x^{*}\right)=\xi$. Therefore we seek here an "inner" solution in the neighborhood of $x=x^{*}$. We use the transformation $z=\left(x-x^{*}\right) / \varepsilon$ in (5) to obtain
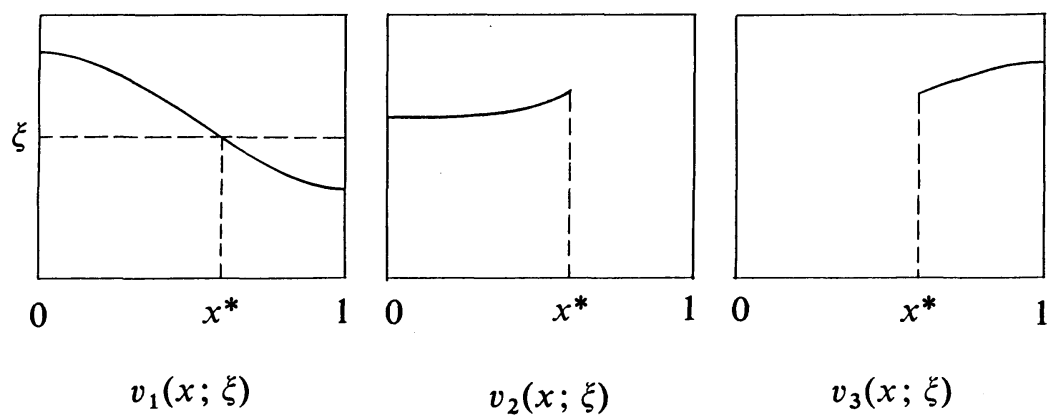

Figure 3A. Spatial distributions of $v_{l}(x ; \xi)$ for suitable $\xi(i=1,2,3)$. 


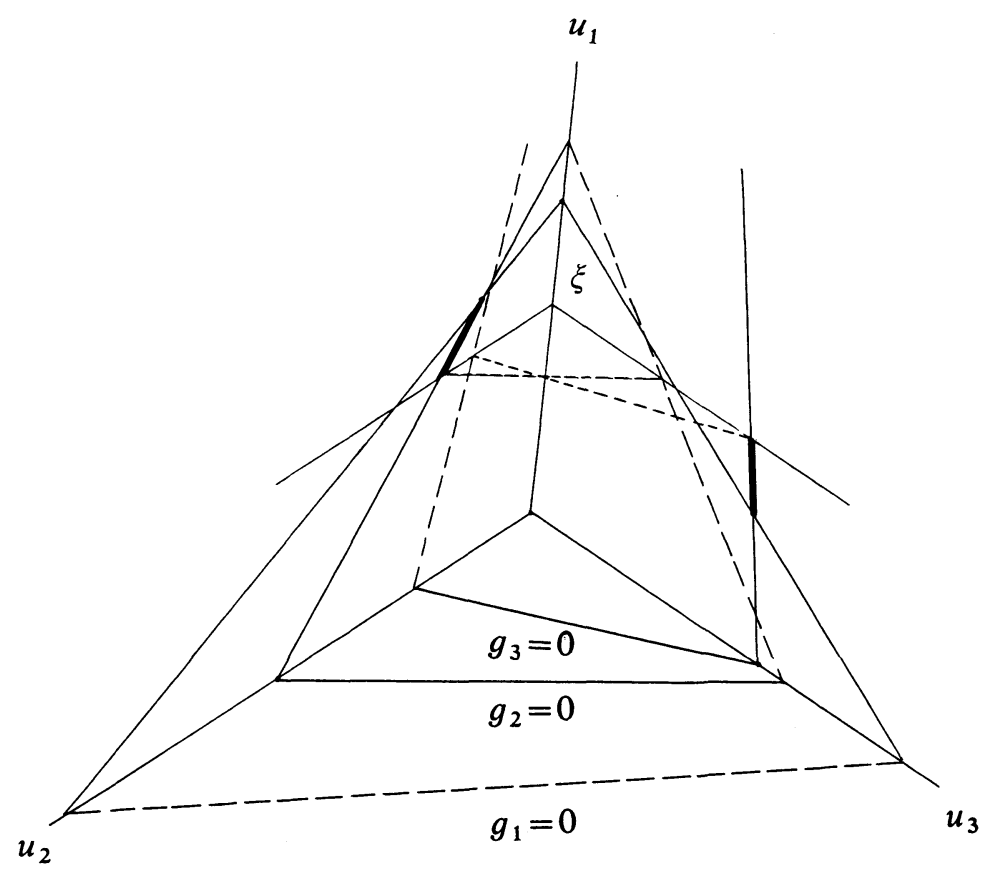

Figure 3B. Bold lines correspond to the outer solution.

$$
\left\{\begin{array}{l}
0=\frac{d^{2} u_{1}}{d z^{2}}+\varepsilon^{2}\left(r_{1}-a_{11} u_{1}-a_{12} u_{2}-a_{13} u_{3}\right) u_{1}, \\
0=\frac{d^{2} u_{2}}{d z^{2}}+\left(r_{2}-a_{21} u_{1}-a_{22} u_{2}-a_{23} u_{3}\right) u_{2}, \\
0=d \frac{d^{2} u_{3}}{d z^{2}}+\left(r_{3}-a_{31} u_{1}-a_{32} u_{2}-a_{33} u_{3}\right) u_{3}
\end{array} .\right.
$$

By putting $\varepsilon=0$ and then $u_{1}(z)=v_{1}\left(x^{*}\right)=\xi$, we obtain a 2-component system with respect to $u_{2}$ and $u_{3}$, which is an approximation to (12):

$$
\left\{\begin{array}{l}
0=\frac{d^{2} U_{2}}{d z^{2}}+\left(R_{2}(\xi)-a_{22} U_{2}-a_{23} U_{3}\right) U_{2} \\
0=d \frac{d^{2} U_{3}}{d z^{2}}+\left(R_{3}(\xi)-a_{32} U_{2}-a_{33} U_{3}\right) U_{3}
\end{array} \quad z \in \mathbf{R},\right.
$$

where $R_{i}(\xi)=r_{i}-a_{i 1} \xi(i=2,3)$. The boundary conditions at $z= \pm \infty$ are specified as

$$
\left\{\begin{array}{l}
U_{2}(-\infty)=R_{2}(\xi) / a_{22} \\
U_{3}(-\infty)=0
\end{array} \text { and } \begin{array}{l}
U_{2}(+\infty)=0 \\
U_{3}(+\infty)=R_{3}(\xi) / a_{33} .
\end{array}\right.
$$


We must study the existnce of a nonnegative solution $\left(U_{2}(z), U_{3}(z)\right)$ to the problem (13), (14).

Here we assume

$$
\frac{a_{21}}{a_{31}}>\frac{r_{2}}{r_{3}}
$$

which implies that the surfaces $r_{2}-a_{21} u_{1}-a_{22} u_{2}-a_{23} u_{3}=0$ and $r_{3}-a_{31} u_{1}-$ $a_{32} u_{2}-a_{33} u_{3}=0$ intersect in $\mathbf{R}_{+}^{3}=\left\{\left(u_{1}, u_{2}, u_{3}\right) \mid u_{i} \geqq 0(i=1,2,3)\right\}$. That is, two lines $R_{2}(\xi)-a_{22} u_{2}-a_{23} u_{3}=0$ and $R_{3}(\xi)-a_{32} u_{2}-a_{33} u_{3}=0$ intersect in $\mathbf{R}_{+}^{2}=$ $\left\{\left(u_{2}, u_{3}\right) \mid u_{2} \geqq 0, u_{3} \geqq 0\right\}$ for each $\xi$ satisfying $\bar{u}_{1}(23 \backslash 2)<\xi<\bar{u}_{1}(23 \backslash 3)$. Thus, we find that as $\xi$ increases, the kinetic system of (13) takes the form of a monostable system $\left(0<\xi<\bar{u}_{1}(23 \backslash 2)\right)$, a bistable one $\left(\bar{u}_{1}(23 \backslash 2)<\xi<\bar{u}_{1}(23 \backslash 3)\right)$ and then a monostable one $\left(\bar{u}_{1}(23 \backslash 3)<\xi\right)$ again.

THEOREM 2. There exists a value $\xi^{*}$ satisfying $\bar{u}_{1}(23 \backslash 2)<\xi^{*}<\bar{u}_{1}(23 \backslash 3)$ such that the problem (13), (14) has a solution $\left(U_{2}\left(z ; \xi^{*}\right), U_{3}\left(z ; \xi^{*}\right)\right)$. (Figure 4).

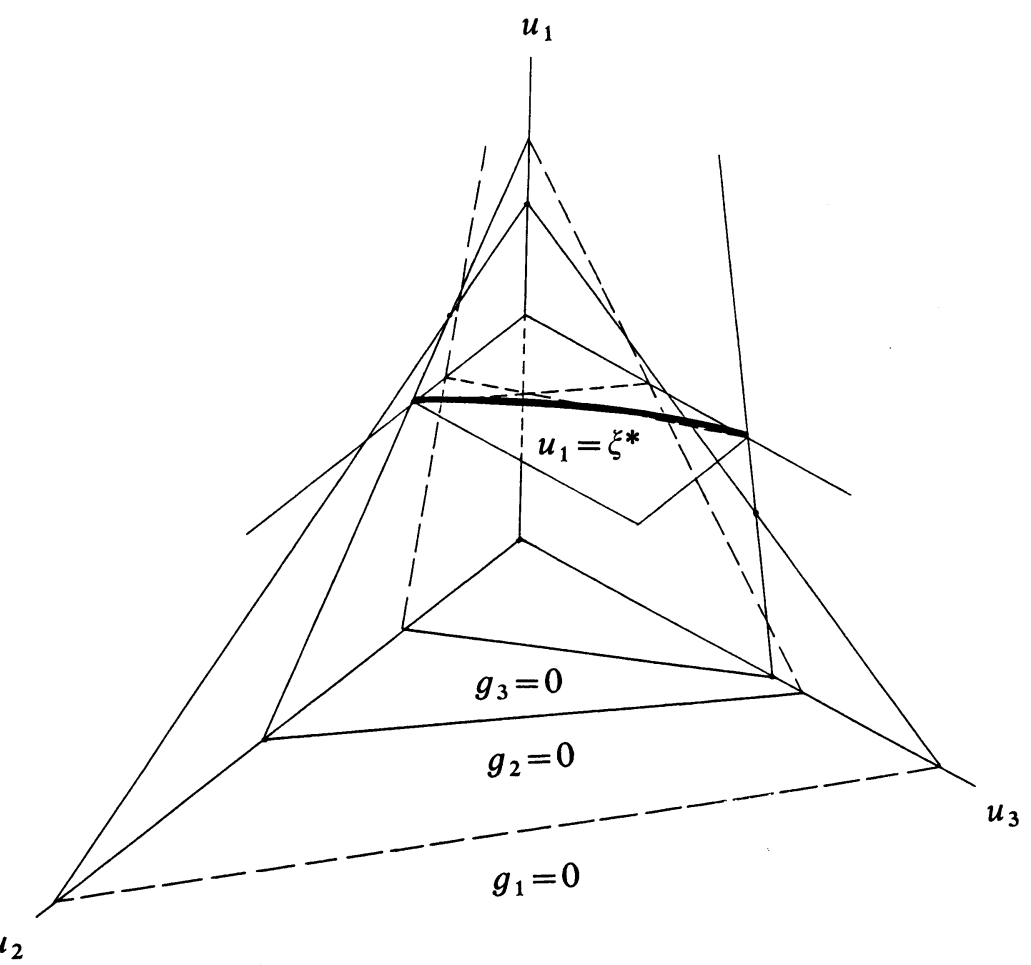

Figure 4. Bold line corresponds to the inner solution. 
Proof: This is a consequence of Theorem A2 in the Appendix, with the following notational changes: subscripts 1 in that theorem are to be replaced by 3 for the present context; $r_{1}$ is to be $R_{3} / d, r_{2}$ is $R_{2}$, and $a_{1 j}$ is $a_{3 j} / d$.

RemarK 1. Theorem 2 does not show explicitly the value of $\xi^{*}$. However, when $a_{22}=a_{33}$ and $a_{32}=a_{23}$, then $\xi^{*}=\left(r_{2}-r_{3}\right) /\left(a_{21}-a_{31}\right)$ (Gardner [4]).

REMARK 2. The uniqueness of such a $\xi^{*}$ is not known, but has been confirmed numerically.

Thus, when we assume

$$
\left(\bar{u}_{1}(23 \backslash 2), \bar{u}_{1}(23 \backslash 3)\right) \subset\left(\bar{u}_{1}(13 \backslash 2), \bar{u}_{1}(12 \backslash 3)\right),
$$

we obtain "outer" solutions $\left(v_{1}\left(x ; \xi^{*}\right), v_{2}\left(x ; \xi^{*}\right), v_{3}\left(x ; \xi^{*}\right)\right)$, as well as "inner" solutions $\left.\left.\left(U_{1}\left(x^{*} ; \xi^{*}\right), U_{2}\left(x-x^{*}\right) / \varepsilon ; \xi^{*}\right), U_{3}\left(x-x^{*}\right) / \varepsilon ; \xi^{*}\right)\right)$, where $\xi^{*}$ is determined by Theorem 2 and $x^{*}$ is found from $v_{1}\left(x^{*} ; \xi^{*}\right)=\xi^{*}$.

The final task is to obtain a solution of the original problem (5), (6) when $\varepsilon$ is sufficiently small but nonzero which corresponds to the "outer" solution of (7), (8) and the "inner" solution of (13), (14) constructed above. Unfortunately we have not yet been able to prove the existence of this solution. We show, however, numerical experiments to supplement the analysis given in this paper, thus further confirming the existence of solutions exhibiting spatial segregation between two of the species (Figure 5).

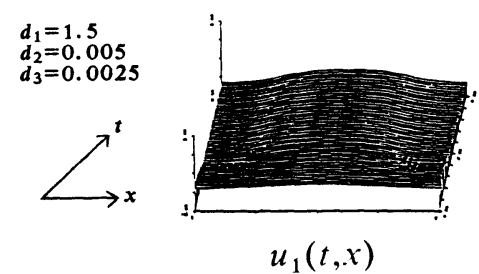

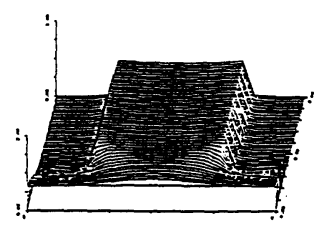

$u_{2}(t, x)$

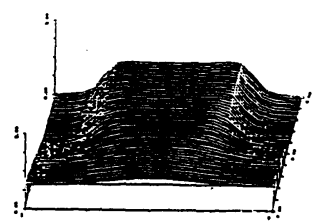

$u_{2}(t, x)$

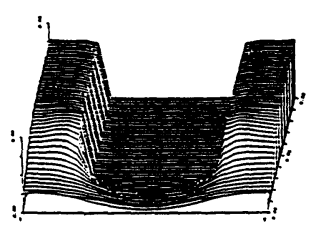

$u_{3}(t, x)$

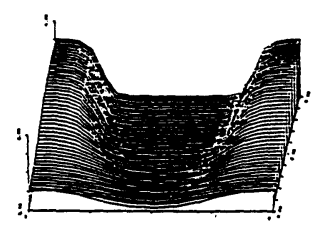

$u_{3}(t, x)$

Figure 5. Spatial distributions of $\left(u_{1}, u_{2} . u_{3}\right)$. 


\section{Concluding remark}

We have discussed non-constant steady state solutions of a three component system of competition and diffusion. In this system, we have assumed that species 1 and 2, as well as 1 and 3, can coexist, but 2 and 3 are of exclusive interaction, that is, either 2 or 3 becomes extinct. Thus, a singular perturbation technique constructs non-constant steady state solutions exhibiting a striking segregated pattern in the species when species 1 diffuses fast but 2 and 3 diffuse slowly. The argument presented here is also valid in the situation where species 2 and 3 coexist, but 2 eliminates 1 as well as 3 .

\section{Appendix.}

Consider the system

$$
\left\{\begin{array}{l}
\frac{d^{2} u}{d x^{2}}+u f(u, v)=0 \\
\frac{d^{2} v}{d x^{2}}+v g(u, v)=0
\end{array}\right.
$$

where $f=r_{1}-a_{11} u-a_{12} v$ and $g=r_{2}-a_{21} u-a_{22} v$. (All of the following extends easily to a much wider class of functions $(f, g)$. We shall not pursue that generality.)

Definition. The pair $(f, g)$ of this form with $r_{i}>0$ and $a_{i j}>0(i, j=1,2)$ is said to belong to class $\mathscr{A}$ if $r_{2} / r_{1}>a_{22} / a_{12}$.

For $(f, g) \in \mathscr{A}$, we shall be concerned with conditions under which there exists a solution of (A1) for $x \in \mathbf{R}$ satisfying

$$
(u, v)(-\infty)=\left(0, r_{2} / a_{22}\right),(u, v)(+\infty)=\left(r_{1} / a_{11}, 0\right) .
$$

With no loss of generality we shall assume, for simplicity, that $r_{2}=a_{22}$ and $r_{1}=$ $a_{11}$. This may always be achieved by rescaling $u$ and $v$. Written as a system of 4 first order equations, (A1) becomes

$$
\left\{\begin{array}{c}
\dot{u}=p, \dot{p}=-u f(u, v) \\
\dot{v}=q, \dot{q}=-v g(u, v)
\end{array}\right.
$$

with $(f, g) \in \mathscr{A}$, where $\cdot=\frac{d}{d x}$. Solutions of the system will be denoted by $X(x)$ where $x=(u, p, v, q)$. Clearly the points $A=(0,0,1,0)$ and $B=(1,0,0,0)$ are rest points. Our main question will be whether there exist trajectories con- 
necting them. The projection of $X(x)$ onto the $(u, v)$ plane will be denoted by $U(x)$, where $U=(u, v)$.

Proposition 1. The rest point $A$ has a local two-dimensional unstable manifold $M$ which may be parameterized by points in the $(u, v)$ plane in $a$ neighborhood of $(0,1)$. More precisely, to each such point $(u, v) \neq(0,1)$, there corresponds exactly one trajectory on $M$, and there exist no other trajectories issuing from $A$. The trajectories themselves may be parameterized by a single real parameter.

Proof. The system (A3), linearized about $A$, can be written as

$$
\dot{\bar{x}}=H \bar{x},
$$

where $H$ has two positive eigenvalues $\lambda_{1}=\sqrt{a_{12}-r_{1}}$ and $\lambda_{2}=\sqrt{r_{2}}$ and two negative ones. The unstable manifold for (A4) is the plane

$$
\bar{p}=\lambda_{1} \bar{u}, \bar{q}=\frac{a_{21}}{\lambda_{1}+\lambda_{2}} \bar{u}+\lambda_{2} \bar{v} .
$$

Its trajectories, projected on the $(\bar{u}, \bar{v})$ plane, are the solutions of

$$
\frac{\bar{q}}{\bar{p}}=\frac{d \bar{v}}{d \bar{u}}=\gamma+\mu \frac{\bar{v}}{\bar{u}} \quad \text { with } \quad \gamma=\frac{a_{21}}{\lambda_{1}\left(\lambda_{1}+\lambda_{2}\right)} \quad \text { and } \quad \mu=\frac{\lambda_{2}}{\lambda_{1}}
$$

and can therefore easily be investigated. For $\varepsilon>0$, they cross transversally the line segment

$$
l_{\varepsilon}: \bar{u}=\varepsilon(1-s), \bar{v}=-a \varepsilon s(0 \leqq s \leqq 1),
$$

provided that $a>\gamma$. Therefore $s$ is a parameter for those trajectories entering the quarter-plane $\{\bar{u} \geqq 0, \bar{v} \leqq 0\}$ which cross $l_{\varepsilon}$; the rest cross the $\bar{u}$-axis between 0 and $\varepsilon$, and can be parametrized by the point at which they do so.

Because of this transversality, if we define the similar segment in the $(u, v)$ plane:

$$
l_{\varepsilon}: u=\varepsilon(1-s), v-1=-a \varepsilon s(0 \leqq s \leqq 1)
$$

for small enough $\varepsilon$, then $s$ can be used to parameterize the trajectories on $M$ whose projections cross $l_{\varepsilon}$ from left to right or top to bottom. We denote these trajectories by $X_{s}(x)=\left(u_{s}(x), p_{s}(x), v_{s}(x), q_{s}(x)\right)$, and their projections by $U_{s}(x)$. The latter are pictured in a neighborhood of $A$ in Figure 6.

We are concerned with elucidating conditions under which, for some $s, X_{s}$ is a connection from $A$ to $B$. We shall denote by $Q$ the square $Q=[0,1] \times$ $[0,1]$ in the $(u, v)$ plane.

We shall need to account for sign changes of $p$ and $q$ along $X_{s}$ : 


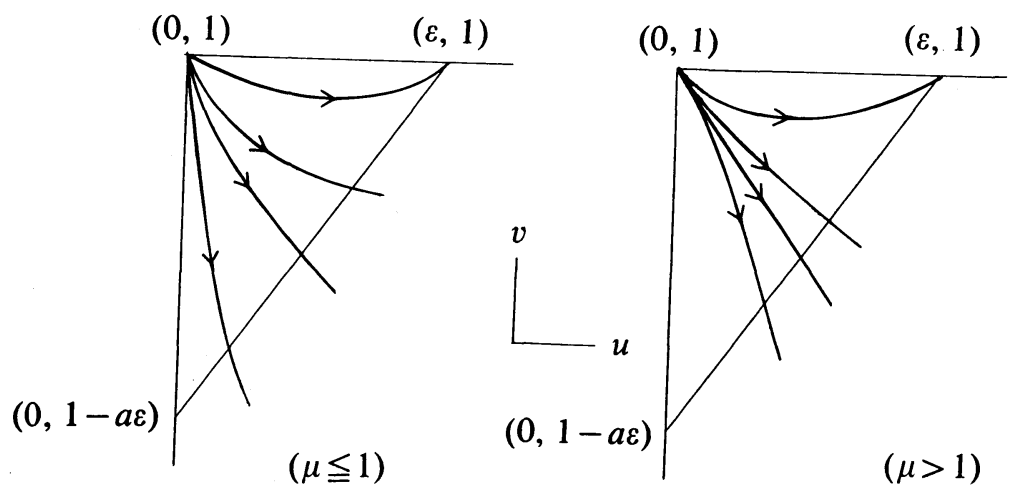

Figure 6. Projection of Trajectories of (A3) onto $(u, v)$-plane.

Definition. The point $U$ is a turning point for $X_{s}$ if it lies on the trajectory $U_{s}$, and if $p_{s}$ or $q_{s}$ vanishes there. (If both $p_{s}$ and $q_{s}$ vanish at that point, it is counted twice in the following proposition.)

Proposition 2. If $U_{s}$ has two or more turning points before leaving $Q$, then the first two are well defined and isolated from the rest. Immediately after the second, $p_{s}<0$ and $g_{s}>0$.

Proof. If $p_{s}\left(x_{1}\right)=0$ for some $x_{1}$, it cannot be true that $\dot{p}_{s}\left(x_{1}\right)=0$, for otherwise $u(x) \equiv u_{s}\left(x_{1}\right), p(x) \equiv 0$ would be a solution of the first two equations in (A3), and by uniqueness of the initial value problem for that subsystem $(v(x)$ being considered known), would equal the first two components of $X_{s}(x)$. This is impossible because our construction does not allow $u$ to be constant. Therefore, $p$ or $q$ (or both) changes sign at each turning point.

Each fixed trajectory $X_{s}$ has $p>0, q<0$ near to $(0,1)$. Suppose $p$ changes sign at the first turning point, and $q$ does not. Since $\dot{p}=-u f<0$ there, that point must lie where $f>0$, i.e. below the line $f=0$. Since $q>0$, the trajectory stays below at least until the second turning point. Therefore $p$ cannot change sign at the second point, since $\dot{p}$ would have the wrong sign, and it must be $q$ that changes. The other possible cases are analyzed in the same way. In particular, although the first two turning points may coincide, the first three may not. This completes the proof.

Proposition 3. If $U_{s}$ does not exit $Q$ and does not have more than one turning point, then it has none, and $X_{s}$ is a connection to $B$.

Proof. There being at most one turning point, $u_{s}(x)$ and $v_{s}(x)$ are eventually monotone, so $X_{s}(x)$ must approach a rest point as $x \rightarrow \infty$. There are four rest points $A, B, O=(0,0,0,0)$ and $E=(\hat{u}, 0, \hat{v}, 0)$ where $(\hat{u}, \hat{v})$ is the intersection 
point of $f=g=0$. The proof consists in eliminating all except $B . A$ is excluded because it would require two turning points. We show why $E$ is also excluded: the argument for $O$ is similar. If $U_{s} \rightarrow(\hat{u}, \hat{v})$ as $x \rightarrow \infty$, the approach must be monotone. It cannot be from the left and above $f=0$, because then $p>0$ and $\dot{p}=-u f>0$, contradicting the requirement that $p \rightarrow 0$. Similarly it can not approach from above, but remaining below $g=0$. This excludes all approaches from above left. Approaches from below left are impossible because on the approach, we would have $q>0$. Since only one sign change was allowed, it must have been $q$, and the turning point must have been below $f=0$ and $g=0$. But that is impossible because $\dot{q}$ would have the wrong sign there. The other directions of approach may be eliminated for similar reasons. This completes the proof.

Definition: $\mathscr{A}_{0} \subset \mathscr{A}$ is the class of functions $(f, g)$ such that for some $s \in(0,1), U_{s}$ has two or more turning points before leaving $Q$.

Proposition 4. Suppose $(f, q) \notin \mathscr{A}_{0}$, and there is no monotone connection, $A$ to $B$. Then every $U_{s}$ exits $Q$ transversally.

Proof. By Proposition 3, $U_{s}$ must exit $Q$ before its second turning point (if it has one). If it exits through the interior of the bottom of $Q$, then $q \leqq 0$ just before and after exit; if $q=0$ at exit, then $\dot{q}=0$ there, and the last two equations of (A3) would attain a rest point at exit, which is impossible. Therefore $q<0$. Similarly exit through the interior of the right side can not be achieved unless $p>0$ there. The other cases are handled in the same way.

Proposition 5. $\mathscr{A}_{0}$ is an open set in the space of parameters $r_{i}$ and $a_{i j}$ $(i, j=1,2)$.

Proof. Consider a trajectory $X_{s}$ with two turning points. The proof of Proposition 4 also shows that sign change of $p_{s}$ can not occur on the lateral sides of $Q$, nor those of $q_{s}$ on the top or bottom. If $p_{s}$ changes sign before $q_{s}$, it must therefore do so in the interior of $Q$, and then the second turning point must be in the interior or on the left side. The latter is excluded because $\dot{q}_{s}=-v_{s} g<0$ there. A similar argument holds if the order is reversed. Therefore, both turning points occur in the interior. Now it is easy to see that the trajectory $X_{s}$ with $s$ fixed, depends continuously on the parameters of the equation. It therefore continues to have two turning points before exiting $Q$ when the parameters are changed by a small amount.

THEOREM A1. Let the family $(f, q)_{r} \in \mathscr{A}$ depend continuously on the real parameter $r$. Assume $(f, g)_{r} \in \mathscr{A}_{0}$ for $r=0$, but not for all positive $r$. Let $r^{*}=\sup \left\{\bar{r}:(f, g)_{r} \in \mathscr{A}_{0}\right.$ for $\left.0 \leqq r \leqq \bar{r}\right\}$. Then $A$ is connected to $B$ for $r=r^{*}$. 
Proof. Our trajectories now depend on two parameters, that is, $U_{s, r}$. Let $\left\{r_{n}\right\}$ be a sequence with $r_{n} \uparrow r^{*}$, and let $s_{n}$ be such that $U_{s_{n}, r_{n}}$ has two turning points before leaving $Q$. By possibly taking a subsequence, we may assume that $s_{n} \rightarrow s^{*}$ as $n \rightarrow \infty$. Now $s^{*} \in(0,1)$, because it is easily seen that any trajectory $U_{s, r}$ with $s$ in a neighborhood of 0 or of 1 does not have two turning points before leaving $Q$. Consider the trajectory $X_{s^{*}, r^{*}}$ By Proposition 5, $(f, g)_{r^{*}} \notin \mathscr{A}_{0}$, hence by Proposition 4, either there exists a connection for $r=r^{*}$, or every trajectory $U_{s, r^{*}}$ leaves $Q$ transversally, before experiencing two turning points. The proof of the theorem consists in excluding the second case. That case would imply, in particular, that $U_{s^{*}, r^{*}}$ exits transversally, and that while $U_{s^{*}, r^{*}}(x) \in Q$, either $p$ or $q$ remains of one sign and is bounded away from zero near the exit point. By continuity of trajectories with respect to parameters, $X_{s^{*}, r^{*}}$ can be approximated by portions of the trajectories $X_{s_{n}, r_{n}}$ for large $n$. Therefore for such $n$, the $U_{s_{n}, r_{n}}$ also exit transversally before experiencing a second turning point. This contradicts their construction and finishes the proof.

Corollary to Proposition 4. If $(f, g) \notin \mathscr{A}_{0}$, then there exists a monotone trajectory which attains or approaches $(1,0)$. If, in addition, no connection exists, then any given point on the bottom or right side of $Q$ is attained by some trajectory $U_{s}$.

Proof: If there exists a monotone connection, we are done. If not, Proposition 4 shows that every $U_{s}$ exits transversally. For small $s$, the exit point is on the top of $Q$. For $s=1$ the trajectory lines on the $v$ axis and the exit point is the origin. By transversality, a standard argument shows that the exit point depends continuously on $s$. Therefore the range of exit points must include every point between those for $s$ near 0 and $s$ near 1 , proceeding either clockwise or counter. But $(0,1)$ is not an exit point (it would require two turning points), so we proceed clockwise, and cover all of the sides indicated.

For the following proposition, we divide $Q$ into four regions

$$
\begin{gathered}
\mathrm{I}=\{f<0<g\} \cap Q, \quad \text { II }=\{f>0, g>0\} \cap Q, \\
\text { III }=\{g<0<f\} \cap Q, \quad \text { IV }=\{f<0, g<0\} \cap Q .
\end{gathered}
$$

For a monotone trajectory $U_{s}$ from $(0,1)$ to $(1,0)$, we denote

$$
\begin{aligned}
& \Gamma=\left\{U_{s}(x)\right\} \cap Q, \\
& J_{I f}=\left|\int_{\Gamma \cap \mathrm{I}} u f(u, v) d u\right|, J_{I g}=\left|\int_{\Gamma \cap \mathrm{I}} v g(u, v) d v\right|
\end{aligned}
$$

and similarly for $J_{\text {II } f^{\prime}}$ etc.

Proposition 6. Let there exist a monotone trajectory $U_{s}$ approaching or 
attaining (1,0). Then the following estimates hold, where the symbols $c$ and $C$ denote positive constants depending only on lower and upper bounds for the constants $r_{i}, a_{i j}(i, j=1,2)$, and $a$, where we define $a=$ the length of $\partial \mathrm{III} \cap\{v=0\}$. They do not represent the same constants in each instance. Here $b$ is the diameter of the region $I$.

(a) $J_{I f}+J_{I g} \leqq C b$;

(b) $J_{\mathrm{IV} g} \geqq c J_{\mathrm{IV} f}-C b$;

(c) the inequality $J_{\mathrm{IIf} f}+J_{\mathrm{IV} f} \equiv s<c$ implies $J_{\mathrm{II} g}+J_{\mathrm{IV} g}>c$ and $J_{\mathrm{II} g} \leqq$ $C(s+b)$

(d) $J_{I f}+J_{\mathrm{IV} f} \geqq J_{\mathrm{II} f}+J_{\mathrm{III} f}$;

(e) $J_{\mathrm{I} g}+J_{\mathrm{II} g} \geqq J_{\mathrm{II} g}+J_{\mathrm{INg}}$.

Proof: (a) follows immediately from the fact that the length of $\Gamma \cap I$ is bounded by $2 b$.

(b) In the limiting case $I=\phi$, we have $v g \rightarrow c u f$ in $\mathrm{IV}$, from which the result follows in that case with $C=0$. When $I \neq \phi$, this inequality is violated only near the intersection $\partial I \cap \partial \mathrm{III}$, which has length $\leqq b$.

(c) The following sketched argument can easily be quantified. The intergral $\int_{\Gamma \cap(\mathrm{IIIN)})}|u f| d u=J_{\mathrm{IIf} f}+J_{\mathrm{IV} f}$ can be made small only by requiring that $\Gamma \cap$ (III U IV) lie mostly in some small enough neighborhood of $\{f=0\}$, the size of the neighborhood depending on a lower bound for $a$. But lying in this neighborhood forces $\left|\int v g d v\right|$ to be larger than some minimum amount, since $|v g|$ is not also small in that neighborhood. It, together with the monotoncity of $\Gamma$, also forces $\Gamma \cap \mathrm{II}$ to lie near $\partial \mathrm{I} \cap \partial \mathrm{II}$, hence the final estimate in (c).

(d) Multiply the second equation of (A3) by $p$ and integrate from $-\infty$ until $(1,0)$ is attained. We find that the value of $p$ at $(1,0)$ satisfies

$$
\frac{1}{2} p^{2}=-\int_{\Gamma} u f(u, v) d u \geqq 0 \text {. }
$$

The integral appearing here is equal to $-J_{\mathrm{I} f}-J_{\mathrm{IV} f}+J_{\mathrm{II} f}+J_{\mathrm{II} f}$, hence (d).

(e) is proved in the same manner.

For purpose of the following theorem, we remove the restriction $r_{2} / a_{22}=$ $r_{1} / a_{11}=1$. Proposition 6 is clearly valid without it.

TheOREM A2. Let the coefficients $r_{i}>0$ and $a_{i j}>0(i, j=1,2)$ depend continuously on a real parameter $\xi$ for $\xi$ in some interval $\Lambda$. Assume that for all $\xi \in \Lambda$

$$
\frac{a_{22}}{a_{12}}<\frac{a_{21}}{a_{11}}
$$


Assume that for some $\xi \in \Lambda, r_{2} / r_{1} \leqq a_{22} / a_{12}$, and for another value, $r_{2} / r_{1} \geqq$ $a_{21} / a_{11}$. Then for some $\xi \in \Lambda$, there exists a solution of (A1), (A2).

Proof. For values of $\xi$ such that $r_{2} / r_{1} \geqq a_{21} / a_{11}$, we have that $(f, g) \in \mathscr{A}$, but $\notin \mathscr{A}_{0}$. To see the latter, observe from the proof of Proposition 2 that if a trajectory has two turning points, they must lie in the region III where $f>0$ and $g<0$. But the assumed inequalities imply that region is empty.

On the other hand, by continuous dependence on $\xi$, there are values of $\xi$ for which $a_{22} / a_{12}<r_{2} / r_{1}<a_{21} / a_{11}$ and $r_{2} / r_{1}$ is arbitrarily close to $a_{22} / a_{21}$. When it is close, the size of $I$ is small, the parameter a, remaining bounded away from zero. For sufficiently small $b$, we show $(f, g) \in \in \mathscr{A}$. This, together with Theorem A1 will establish the desired result. It suffices to show there exists no monotone trajectory attaining or approaching $\left(r_{1} / a_{11}, 0\right)$. Suppose there were. A straightforward calculation shows that $\Gamma$ must be concave upward in IV and downward in II. It follows from this and the fact that $\Gamma$ must reach $\left(r_{1} / a_{11}, 0\right)$ that either $\Gamma \cap$ II or $\Gamma \cap I V$ must be empty. Suppose it is the former. For some $\delta>0$, let $\Lambda_{0}$ be the set of $\xi$ for which $a_{22} / a_{12}<r_{2} / r_{1}<a_{22} / a_{12}+\delta$. In the following, the constants $c$ and $C$ are uniformly bounded from below and above for $\xi \in \Lambda_{0}$, and do not necessarily denote the same constant, even within a single context. From (a) in Proposition 6, we have $J_{\mathrm{I} f}+J_{\mathrm{I} g} \leqq C b$, and from (e), $C b \geqq J_{\mathrm{I} g} \geqq J_{\mathrm{IIg} g}+J_{\mathrm{IV} g}$. Hence if $b$ is small enough, the inequality in (c) regarding $J_{\mathrm{IIg}_{g}}+J_{\mathrm{IVg}}$ is violated, hence $J_{\mathrm{III} f}+J_{\mathrm{IV} f} \geqq c$. From this and the fact (d) that $J_{\mathrm{I} f}+J_{\mathrm{IV} f} \geqq J_{\mathrm{III} f}$, we have $J_{\mathrm{I} f}+2 J_{\mathrm{IV} f} \geqq J_{\mathrm{III} f}+J_{\mathrm{IV} f} \geqq c$, so $J_{\mathrm{IV} f} \geqq \frac{1}{2}(c-C b)$. The same type of inequality also holds (b) for $J_{\mathrm{IV} g}: J_{\mathrm{IV} g} \geqq \frac{c}{2}(c-C b) \geqq c^{2} / 4$ (for $b$ small enough). From (e), this implies $J_{\mathrm{I} g} \geqq c^{2} / 4$, which for small $b$ contradicts the fact that $J_{\mathrm{I} g} \leqq C b$.

The other case is when $\Gamma \cap \mathrm{IV}=\phi$. Again, we have $J_{\mathrm{I} f} \leqq C b$. From (d), we obtain $C b>J_{\mathrm{I} f} \geqq J_{\mathrm{II} f}=J_{\mathrm{II} f}+J_{\mathrm{IV} f}$, so the hypothesis of (c) is fulfilled for small b. Hence

$$
J_{\mathrm{II} g} \leqq C(b+s) \leqq C b .
$$

But this contradicts (e), whose left side is bounded by $C b$, and right side (by (c)) is bounded from below. Therefore, no such $\Gamma$ can exist. This completes the proof.

\section{References}

[1] Crombie, A. C., On intraspecific and interspecific competition in larvae of graminivorous insects. J. Exp. Biol., 20, 135-151 (1944).

[2] de Mottoni, P., Qualitative analysis for some quasilinear parabolic systems. Warsaw PAN Mathematics Institute. Preprint 171 (1979).

[3] Evans, G. T., Diffusive structure: Counterexamples to any explanation? J. Theor. 
Biol., 82, 313-315 (1980).

[ 4 ] Gardner, R. A., Large amplitude patterns for two competing species, Technical Summary Report \#2090, MRC, Univ. of Wisconsin-Madison, (1980).

[5] Kishimoto, K., Instability of non-constant equilibrium solutions of a system of competition-diffusion equations. J. Math. Biol. 13, 105-114 (1981).

[6] Kishimoto, K., The diffusive Lotka-Volterra system with three species can have a stable non-constant euqilibrium solutions. J. Math. Biol. 16, 103-112 (1982).

[7] Kishimoto, K., Mimura, M. and Yoshida, K., Stable spatio-temporal oscillations of diffusive Lotka-Volterra systems with three or more species, J. Math. Biol. 18, 213-221 (1983).

[ 8 ] Kishimoto, K. and Weinberger, H., The spatial homogeneity of stable equilibria of some reaction-diffusion systems on convex domains, J. Differential Equations 58, 15-21 (1985).

[9] Levin, S. A., Nonuniform steady state solutions of reaction-diffusion equations: applications to ecological pattern formation. In: Pattern formation by dynamical systems and pattern recognition, H. Haken ed., Springer-Verlaq, 210-225 (1979).

[10] Matano, H. and Mimura, M., Pattern formation in competition-diffusion systems in nonconvex domains, Publ. RIMS, Kyoto Univ. 19, 1049-1079 (1983).

[11] Mimura, M., Stationary pattern of some density-dependent diffusive systems with competing dynamics. Hiroshima Math. J., 11, 621-635 (1981).

[12] Mimura, M. and Kawasaki, K., Spatial segregation in competitive interaction-diffusion equations, J. Math. Biol., 9, 49-64 (1980).

[13] Schoener, T. W., Resource partitioning in ecological communities, Science 185, 27-39 (1974).

[14] Shigesada, N., Kawasaki, K. and Teramoto, E., Spatial segregation of interacting species, J. Theor. Biol., 79, 83-99 (1979).

[15] Trägardh, I., Entomologiska analyser av torkande tråd. Medd. Skogsförsökasnst., 23, 191-216 (1927).

[16] Turing, A. M., The chemical basis for morphogenesis, Phil. Trans. Roy. Soc. (London) B 237, 37-72 (1952).

Department of Mathematics, Faculty of Science, Hiroshima University and

Department of Mathematics, The University of Arizona 
